\title{
Case Report on Autoimmune Hemolytic Anemia: A Mask of Malignancy!
}

\begin{abstract}
A 10-year-old boy with autoimmune hemolytic anemia (AIHA) was treated at a local setup with repeated blood transfusions and steroids considering an idiopathic etiology. After 7 months of unresponsiveness, the child started developing recurrent fever with night sweats and pain in the abdomen which was diagnosed as tuberculosis on account of granulomatous necrotizing lesion on computed tomography (CT)-guided lymph node biopsy and treated for the same. Four months later, when the child presented to our center in anemic heart failure, work-up with positron emission tomography-CT and excisional biopsy of mesenteric lymph node led to the diagnosis of advanced Hodgkin's lymphoma with B symptoms. AIHA in pediatric age group, unlike in adults, is usually secondary. Our report stresses on a thorough evaluation of AIHA as it can mask a malignancy for a long duration and alter its course if treated with steroids, making the patient resistant to further chemotherapy.
\end{abstract}

Keywords: Autoimmune hemolytic anemia, Hodgkin's lymphoma, tuberculosis

\section{Introduction}

Autoimmune hemolytic anemia (AIHA) of childhood is a very rare disease, with an estimated incidence of 2 per million. ${ }^{[1]}$ It can be idiopathic or secondary to various infections, autoimmune disorders, immunodeficiency diseases, neoplasms, or drugs. ${ }^{[2]}$ Secondary AIHA, when appears solely as initial manifestation, without other signs and symptoms of its associated disease, can be labeled as idiopathic and treated accordingly with steroids. However, steroids can have adverse effects on the underlying disease by either flaring it up or deferring its diagnosis. The infection of tuberculosis can mimic various diseases including malignancies. ${ }^{[3]}$ Our case is an illustration of Hodgkin's lymphoma (HL) presenting at an advanced stage on account of a potentially misleading idiopathic AIHA and tuberculosis-like picture.

\section{Case Report}

A 10-year-old boy presented to us with heart failure as a consequence of severe anemia. His first presentation was to a local doctor 11 months back with symptoms of anemia. With a low hemoglobin and

\footnotetext{
This is an open access journal, and articles are distributed under the terms of the Creative Commons Attribution-NonCommercialShareAlike 4.0 License, which allows others to remix, tweak, and build upon the work non-commercially, as long as appropriate credit is given and the new creations are licensed under the identical terms.
}

For reprints contact: WKHLRPMedknow_reprints@wolterskluwer.com positive direct antiglobulin test, a diagnosis of idiopathic AIHA was made, and he was transfused with the most compatible blood and treated with steroids. Three months later, he had a similar episode. Five months later, he developed the third episode of illness. During this episode, he developed additional complaints of on and off fever associated with night sweats. $\mathrm{He}$ also complained of diffuse abdominal pain of 1-month duration. Work-up for pyrexia of unknown origin failed to reveal any etiology. In view of his abdominal pain, computed tomography (CT) scan was done which showed hepatosplenomegaly with multiple enlarged pre- and para-aortic and mesenteric groups of lymph nodes. CT-guided core biopsy of lymph node was reported as necrotizing granulomatous lesion suggestive of tuberculosis. Now with a possible diagnosis of tuberculosis, the patient was started on empirical antitubercular treatment (ATT). When the child presented to our center, he had completed 4 months of ATT with no improvement in his symptoms. He also had a significant weight loss of $5 \mathrm{~kg}$ in the past 6 months. So far, he had no complaints of cough, rash, joint pains, swelling in the neck or any other part of the body, and bleeding manifestations.

How to cite this article: Sampagar A, Patil NG, Bartakke S, Shrividya S. Case report on autoimmune hemolytic anemia: A mask of malignancy! Indian J Med Paediatr Oncol 2020;41:909-12.

\section{Abhilasha Sampagar ${ }^{1}$, Neha Giridhar Patil' Sandip Bartakke ${ }^{2}$, S Shrividya ${ }^{2}$}

${ }^{1}$ Department of Pediatrics, KAHER'S Jawaharlal Nehru Medical College, Belagavi, Karnataka, India, ${ }^{2}$ Department of Hematology, Aditya Birla Memorial Hospital, Pune, Maharashtra, India

Submitted: $22-A p r-2020$ Revised: 04-Aug-2020 Accepted: 23 -Aug-2020 Published: 31-Dec-2020

Address for correspondence: Dr. Abhilasha Sampagar, Pediatric HematologistOncologist, Associate Professor, Department of Pediatrics, KAHER's Jawaharlal Nehru Medical College, Belagavi, Karnataka, India.

E-mail: abhilasha.pedia@gmail. com

Access this article online

Website: www.ijmpo.org

DOI:10.4103/ijmpo.ijmpo_182_20 Quick Response Code:

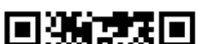


On examination, the child had fever, pallor, body mass index of $12.62 \mathrm{~kg} / \mathrm{m}^{2}$, bilateral pedal edema, loud $\mathrm{S} 1$, gallop rhythm, systolic murmur best heard in the pulmonary area, and fine crepitations in bilateral basal lung fields. Per abdominal examination revealed hepatomegaly with a liver span of $17 \mathrm{~cm}$, smooth surface, rounded margins, and firm in consistency. The spleen was enlarged up to $5 \mathrm{~cm}$ below the left costal margin in the spinoumbilical line.

The considered differential diagnoses were multidrugresistant/disseminated tuberculosis, lymphoma, HIV and autoimmune lymphoproliferative syndrome (ALPS), disseminated histoplasmosis, kala-azar, and hemophagocytic histio-lymphocytosis. Laboratory findings at our center are depicted in Table 1.

Negative results for antinuclear antibodies, deranged immunoglobulins, and double-negative T-cells made the possibilities of connective tissue disorders and ALPS less likely. HIV testing and gastric samples sent for gene expert for tuberculosis were also negative. CT scan of the abdomen and pelvis revealed enlarged liver measuring $20 \mathrm{~cm}$, showing few tiny hypodense nodular lesions, largest measuring approximately $7.0 \mathrm{~mm} \times 6.0 \mathrm{~mm}$ in segment VII, and enlarged spleen measuring $13.0 \mathrm{~cm} \times 4.2 \mathrm{~cm}$ showing multiple hypodense areas diffusely involving the splenic parenchyma.

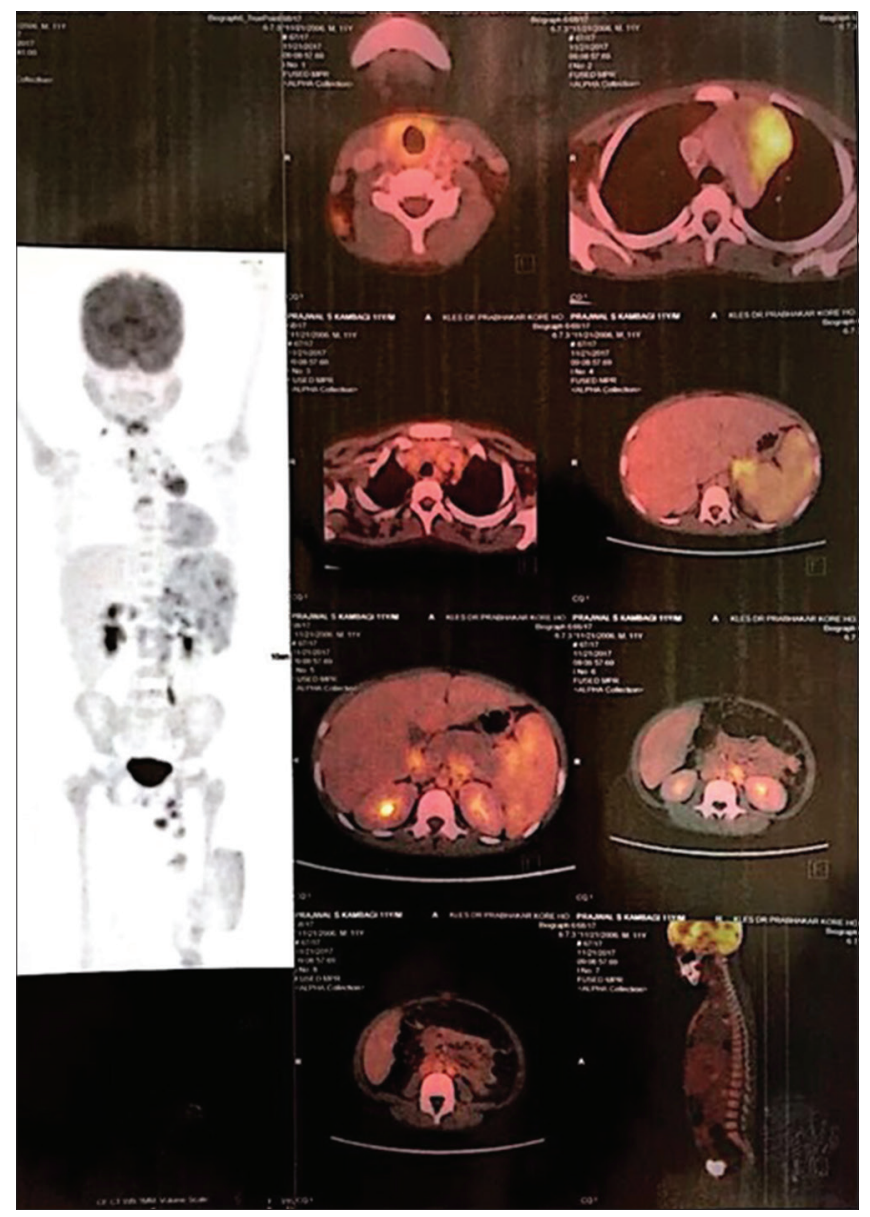

Figure 1: Baseline positron emission tomography-computed tomography
It also revealed multiple enlarged pre- and para-aortic, preand retrocaval, and mesenteric groups of lymph nodes, largest approximately measuring $1.8 \mathrm{~cm} \times 1.6 \mathrm{~cm}$ in the left paraaortic group. CT scan of the thorax showed homogeneously enhancing mass lesion in the perivascular space of the mediastinum, approximately measuring $4.0 \mathrm{~cm} \times 3.0 \mathrm{~cm}$. Positron emission tomography (PET) scan showed increased metabolic uptake in right cervical level IV (SUVmax - 3.7) and V (SUVmax - 2.38) lymph nodes, mediastinal group (SUVmax - 5.11), and retroperitoneal and abdominal group of lymph nodes (SUVmax of porta hepatic - 3.14, aortocaval -2.73 , para-aortic -3.5 , celiac group -3.58 , and splenic hilar - 3.26). There were hypermetabolic hypodense lesions in an enlarged spleen (SUVmax - 3.25). However, an enlarged liver did not show any focal fluorodeoxyglucose (FDG)-avid lesions [Figure 1]. Laparoscopic intra-abdominal excisional lymph node biopsy and immunohistochemistry positive for CD30 and PAX-5 [Figures 2-4] confirmed the diagnosis of classical HL, mixed cellularity type of Stage IV as per Lugano classification.

In addition to supportive care including packed red cell transfusion, the patient was started on chemotherapy as per German protocol, consisting of two cycles of vincristine, etoposide, prednisolone, and adriamycin and four cycles of cyclophosphamide, vincristine, prednisolone, and dacarbazine. A dramatic improvement in symptoms, with a quick rise in hemoglobin levels, and disappearing evidence of hemolysis were noticed after the first cycle of chemotherapy. After two cycles of chemotherapy, the PET-CT showed complete metabolic response with a Deauville score of 1 . He further completed four cycles of chemotherapy and continues to be in remission for the past 2 years. The child has gained $8 \mathrm{~kg}$ of weight in the past 2 years, fever and hepatosplenomegaly have subsided, and the child has never required blood transfusion again. His current hemoglobin is $12.2 \mathrm{~g} / \mathrm{dL}$. Repeat PET scan 3 months back revealed no FDG uptake in lymph nodes or spleen. The child is asymptomatic and on regular follow-up.

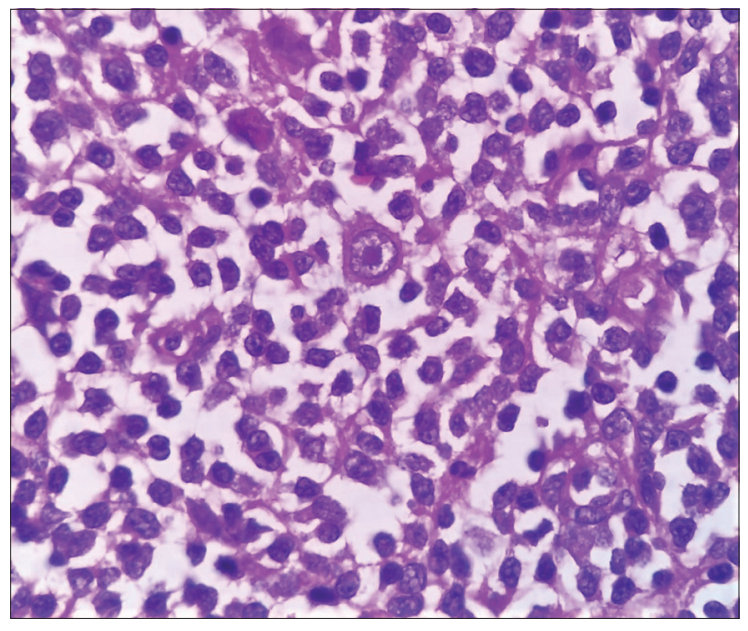

Figure 2: Hodgkin Reed-Sternberg cells 


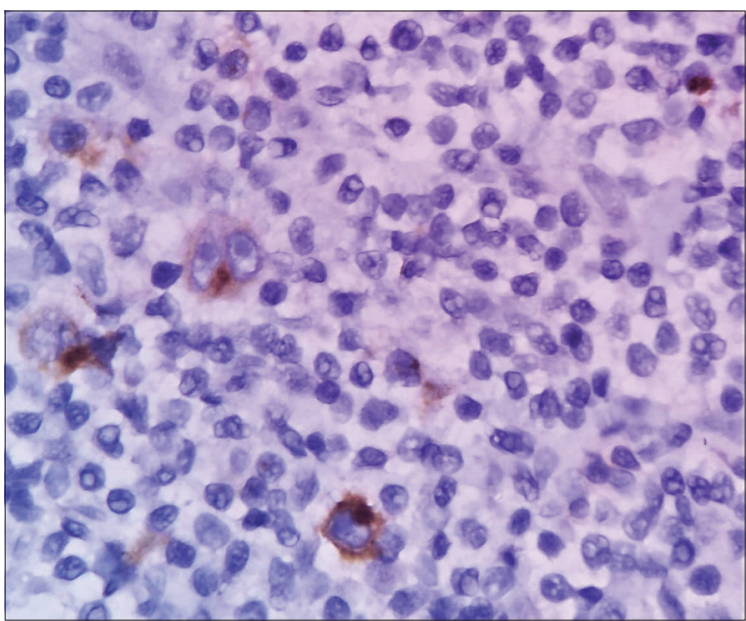

Figure 3: Immunohistochemistry-CD30 positive

\begin{tabular}{lccc}
\hline \multicolumn{4}{c}{ Table 1: Laboratory findings } \\
\hline & $\begin{array}{c}\text { Observed } \\
\text { value }\end{array}$ & Units & $\begin{array}{c}\text { Reference } \\
\text { range }\end{array}$ \\
\hline Hemogram \\
Hemoglobin & 3.4 & $\mathrm{~g} / \mathrm{dL}$ & $11.5-15.5$ \\
MCV & 139.9 & $\mathrm{fl}$ & $77.0-95.0$ \\
Red blood cell count & 0.82 & $10^{6} / \mathrm{micro} \mathrm{L}$ & $4.00-5.10$ \\
Reticulocyte count & 6.0 & $\%$ & $0.5-1.5$ \\
White blood cell count & 6.0 & $10^{3} / \mathrm{micro} \mathrm{L}$ & $5.0-13.0$ \\
Platelet count & 292 & $10^{3} / \mathrm{micro} \mathrm{L}$ & $172-450$ \\
\hline \multicolumn{4}{c}{ Peripheral smear-dimorphic anemia with hemolysis } \\
\hline LDH & 806 & $\mathrm{U} / \mathrm{L}$ \\
Direct Coombs test - 3+ (anti-IgG) & $135-225$ \\
\hline \multicolumn{1}{c}{ Bone marrow report features suggestive of megaloblastic } \\
\multicolumn{4}{c}{ hypercellular marrow } \\
\hline Albumin & 2.8 & $\mathrm{~g}$ & $3.5-5$ \\
Total bilirubin & 5.1 & $\mathrm{mg} / \mathrm{dL}$ & $0.1-1.2$ \\
Direct bilirubin & 0.9 & $\mathrm{mg} / \mathrm{dL}$ & $<0.3$ \\
\hline
\end{tabular}

MCV - Mean corpuscular volume; LDH - Lactate dehydrogenase

\section{Discussion}

$\mathrm{HL}$ is seldom associated with autoimmune conditions such as AIHA, immune thrombocytopenic purpura, and nephrotic syndrome. The reported incidence of AIHA in $\mathrm{HL}$ at $0.2 \%-4.2 \%$ is relatively rare. Reports have shown that AIHA can occur in any stage of HL, most commonly in Stages III and IV of nodular sclerosis type or mixed cellularity type. However, very seldom does AIHA present as an initial symptom of HL and can potentially confound its diagnosis. ${ }^{[4,5]}$ Defective apoptotic pathways in HL lead to excessive accumulation of T-cells which facilitate antibody production by B-cells. These excess antibodies are responsible for autoimmune manifestations of the disease. ${ }^{[6]}$ Contrary to adults, AIHA in pediatric age group is usually secondary. ${ }^{[7]}$ Administration of steroids in a patient of AIHA without looking into its cause can further obscure the diagnosis of possible hidden malignancy such as HL by altering its clinical and

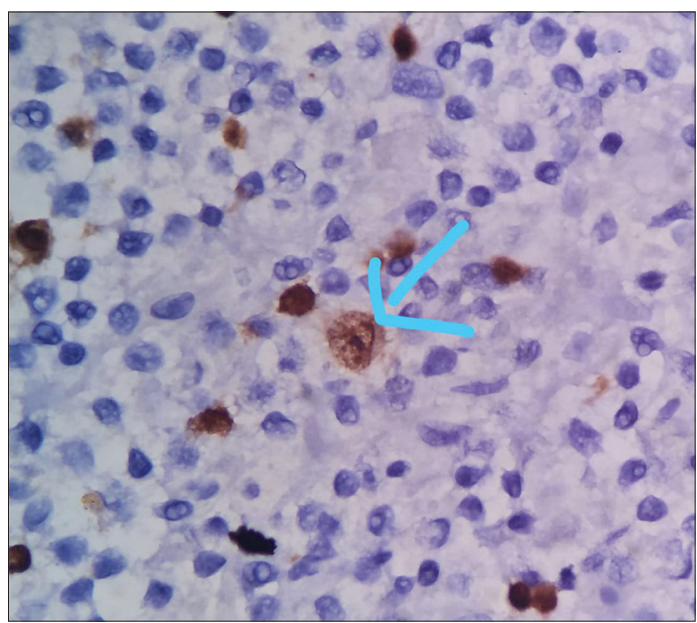

Figure 4: Immunohistochemistry-PAX-5 positive

histopathological picture and making the patient resistant to chemotherapy. ${ }^{\left[{ }^{[8]}\right.}$

Tuberculosis, on the other hand, being great mimicker can present with anemia, fever, night sweats, weight loss, anorexia, hepatosplenomegaly, and elevated LDH, all of which also form signs and symptoms of lymphoma. The two can closely resemble each other on radiology as well as histopathology with similar pictures of necrotizing granulomatous lesion as also seen in few reports, ${ }^{[3]}$ and sometimes, these two entities can coexist. Therefore, with a high index of suspicion, careful pathological evaluation including immunohistochemistry is warranted. Excisional biopsy always stands superior to fine-needle aspiration cytology or core biopsy. ${ }^{[9]}$

The Childhood Hodgkin International Prognostic Score (CHIPS) identifies four independent predictors of event-free survival Stage 4 disease, large mediastinal mass, albumin $(<3.5)$, and fever. The 4-year survival rate drops from $88.5 \%$ for patients with CHIPS $=0 \%-69.2 \%$ for CHIPS $=3 .^{[10]}$ Hence, in a developing country like India with a high burden of tuberculosis, contrary to common practice of overdiagnosis and early initiation of ATT, withholding ATT until exclusion of malignancy and surety of diagnosis, especially in cases presenting as steroid-refractory autoimmune anemias, can not only save the patient from advancing to poorer stage but also from ill-effects of antitubercular drugs for the period of unresponsiveness. Moreover, an AIHA should be thoroughly evaluated for any possible secondary cause before assigning an idiopathic etiology to it.

Our case, a patient with AIHA, with no clinical lymphadenopathy, treated initially with steroids followed by ATT in view of needle core biopsy suggestive of tuberculosis, presenting to us after 11 months of the onset of symptoms in advanced stage of HL reflects the need of a high index of suspicion and thorough evaluation of unexplained manifestations and confounding clinical and 
histopathological presentations, without haste in initiation of empirical treatment to prevent the detrimental effects of delayed diagnosis of a hidden malignancy. Ironically, this case holds its importance not only in being an uncommon diagnosis but also being commonly misdiagnosed in practice.

\section{Declaration of patient consent}

The authors certify that they have obtained all appropriate patient consent forms. In the form, the parent has given his consent for images and other clinical information to be reported in the journal. The parent understands that names and initials will not be published and due efforts will be made to conceal identity, but anonymity cannot be guaranteed.

\section{Financial support and sponsorship}

Nil.

\section{Conflicts of interest}

There are no conflicts of interest.

\section{References}

1. Fan J, He H, Zhao W, Wang Y, Lu J, Li J, et al. Clinical features and treatment outcomes of childhood autoimmune haemolytic anemia: A retrospective analysis of 68 cases. J Pediatr Hematol Oncol 2016; 38:e50-5.

2. Packman $\mathrm{CH}$. The clinical pictures of autoimmune hemolytic anemia. Transfus Med Hemother 2015;42:317-24.

3. Uy AB, Garcia AM, Manguba A, Loyola A. Tuberculosis: The great lymphoma pretender. Int J Cancer Res Mol Mech 2016;2:1-4.

4. Xirox N, Binder T, Anger B, Bohlke J, Heimpel H. Idiopathic thrombocytopenic purpura and autoimmune haemolytic anemia in Hodgkin's disease. Eur J Haematol 1988;40:437-41.

5. Lechner K, Chen YA. Paraneoplastic autoimmune cytopenias in Hodgkin's lymphoma. Leuk Lymphoma 2010;51:469-74.

6. Orkin SH, Nathan DG, Ginsburg D, Look AT, Fisher DE, Lux S. Nathan and Oski's hematology and oncology of infancy and childhood E-book. Philadelphia : Elsevier Health Sciences; 2014.

7. Ladogana S, Maruzzi M, Samperi P, Perrotta S, Del Vecchio GC, Notarangelo LD, et al. AIHA committee of the Italian association of paediatric onco-hematology (AIEOP) diagnosis and management of newly diagnosed childhood autoimmune hemolyticanemia. Recommendations from the red cell study group of the paediatric haemato-oncology Italian association. Blood Transfus 2017;15:259-67.

8. Kan E, Levi I, Benharroch D. Alterations in the primary diagnosis of lymphomas pretreated with corticosteroid agents. Leuk Lymphoma 2011;52:425-8.

9. Allin D, David S, Jacob A, Mir N, Giles A, Gibbins N. Use of core biopsy in diagnosing cervical lymphadenopathy: A viable alternative to surgical excisional biopsy of lymph nodes? Ann R Coll Surg Engl 2016;5:1-3.

10. Schwartz CL, Chen L, McCarten K, Wolden S, Constine LS, Hutchison RE, et al. Childhood Hodgkin International Prognostic Score (CHIPS) predicts event-free survival in Hodgkin lymphoma: Areport from the Children's Oncology Group. Pediatric Blood and Cancer 2017;64:e26278. 